FILE: Coeff-BP.tex, printed: 2018-10-8, 5.00

\title{
ON THE TAYLOR COEFFICIENTS OF A SUBCLASS OF MEROMORPHIC UNIVALENT FUNCTIONS
}

\author{
BAPPADITYA BHOWMIK * AND FIRDOSHI PARVEEN
}

\begin{abstract}
Let $\mathcal{V}_{p}(\lambda)$ be the collection of all functions $f$ defined in the unit disc $\mathbb{D}$ having a simple pole at $z=p$ where $0<p<1$ and analytic in $\mathbb{D} \backslash\{p\}$ with $f(0)=0=f^{\prime}(0)-1$ and satisfying the differential inequality $\left|(z / f(z))^{2} f^{\prime}(z)-1\right|<$ $\lambda$ for $z \in \mathbb{D}, 0<\lambda \leq 1$. Each $f \in \mathcal{V}_{p}(\lambda)$ has the following Taylor expansion:

$$
f(z)=z+\sum_{n=2}^{\infty} a_{n}(f) z^{n}, \quad|z|<p .
$$

In [4, we conjectured that

$$
\left|a_{n}(f)\right| \leq \frac{1-\left(\lambda p^{2}\right)^{n}}{p^{n-1}\left(1-\lambda p^{2}\right)} \quad \text { for } \quad n \geq 3 .
$$

In the present article, we first obtain a representation formula for functions in the class $\mathcal{V}_{p}(\lambda)$. Using this representation, we prove the aforementioned conjecture for $n=3,4,5$ whenever $p$ belongs to certain subintervals of $(0,1)$. Also we determine non sharp bounds for $\left|a_{n}(f)\right|, n \geq 3$ and for $\left|a_{n+1}(f)-a_{n}(f) / p\right|, n \geq 2$.
\end{abstract}

\section{INTRODUCTION}

We shall use following notations throughout the discussion of this article. Let $\mathbb{C}$ be the whole complex plane, $\mathbb{D}:=\{z \in \mathbb{C}:|z|<1\}$ and $\Delta:=\{\zeta \in \mathbb{C}:|\zeta|>1\} \cup\{\infty\}$. Let $\mathcal{A}$ be the class of all analytic functions $f$ defined in $\mathbb{D}$ with the normalization $f(0)=0=f^{\prime}(0)-1$ and $\mathcal{S}=\{f \in \mathcal{A}: f$ is univalent $\}$. Each $f \in \mathcal{S}$ has the following Taylor expansion:

$$
f(z)=z+\sum_{n=2}^{\infty} a_{n}(f) z^{n}, \quad z \in \mathbb{D} .
$$

In the last century, the field of geometric function theory provided many interesting and fascinating facts. One of the main problem of this field was the Bieberbach conjecture which was proposed in the year 1916. This conjecture states that each $f \in \mathcal{S}$ with the expansion (1.1) must satisfy the inequality $\left|a_{n}(f)\right| \leq n$ for all $n \geq 2$. In the year 1985, L. de Branges [5] proved this conjecture. In order to settle the Bieberbach conjecture prior to the effort made by de Branges, many subclasses of $\mathcal{S}$ were introduced that are geometric in nature and the conjecture was being proved for these subclasses. Some of the special subclasses of $\mathcal{S}$ for which this conjecture was settled were the class of convex functions, starlike functions and close to convex functions.

2010 Mathematics Subject Classification. 30C45.

Key words and phrases. Meromorphic functions, Univalent functions, Subordination, Taylor coefficients. 
In [1], Aksentév proved a sufficient condition for the functions of the form

$$
F(\zeta)=\zeta+\sum_{n=0}^{\infty} b_{n} \zeta^{-n}, \quad \zeta \in \Delta,
$$

to be univalent in $\Delta$. This condition enables many authors to consider the following class of functions:

$$
\mathcal{U}(\lambda):=\left\{f \in \mathcal{A}:\left|U_{f}(z)\right|<\lambda \text { for } z \in \mathbb{D}\right\}
$$

where $U_{f}(z):=(z / f(z))^{2} f^{\prime}(z)-1$ and $0<\lambda \leq 1$. It is well-known that each function in $\mathcal{U}(\lambda)$ is univalent and $\mathcal{U}(\lambda) \varsubsetneqq \mathcal{S}$. For a detailed study of the class $\mathcal{U}(\lambda)$ one may go through the articles [6, 8, 9, 10] and references therein. In this note, we consider the meromorphic analogues of the classes $\mathcal{A}, \mathcal{S}$ and $\mathcal{U}(\lambda)$. To this end, let $\mathcal{A}(p)$ be the class which is defined as the collection of functions in $\mathbb{D}$ having a simple pole at $z=p$ where $p \in(0,1)$ and analytic in $\mathbb{D} \backslash\{p\}$ satisfying the normalization $f(0)=0=f^{\prime}(0)-1$. Let $\Sigma(p):=\{f \in \mathcal{A}(p): f$ is univalent $\}$. In [2], we established the following sufficient condition for functions in $\mathcal{A}(p)$ to be univalent:

Theorem A. Let $f \in \mathcal{A}(p)$. If $\left|\mathcal{U}_{f}(z)\right| \leq((1-p) /(1+p))^{2}$ for $z \in \mathbb{D}$, then $f$ is univalent in $\mathbb{D}$.

Motivated by this sufficient condition stated in the above theorem, in [2] we considered the following subclass of $\Sigma(p)$.

Definition 1.1. Let $\mathcal{U}_{p}(\lambda)$ be the family of all functions $f \in \mathcal{A}(p)$ such that $\left|U_{f}(z)\right|<\lambda((1-p) /(1+p))^{2}, z \in \mathbb{D}$ holds for some $0<\lambda \leq 1$.

Interested reader may go through the articles [2] and [3] for many other interesting results for functions in the class $\mathcal{U}_{p}(\lambda)$. We point out here that in [4], we improve the sufficient condition in Theorem A for univalence with replacing the number $((1-p) /(1+p))^{2}$ by 1 and subsequently the following class of functions $\mathcal{V}_{p}(\lambda)$ was introduced:

$$
\mathcal{V}_{p}(\lambda)=\left\{f \in \mathcal{A}(p):\left|U_{f}(z)\right|<\lambda, z \in \mathbb{D}\right\}, \quad \text { for } \lambda \in(0,1] .
$$

In [4], we proved that $\mathcal{U}_{p}(\lambda) \subsetneq \mathcal{V}_{p}(\lambda)$ and discussed many other aspects of this class of functions. Let $\mathcal{B}$ be the class of functions $w$ which are analytic in $\mathbb{D}$ and for $z \in \mathbb{D},|w(z)| \leq 1$. In [4], we proved the following integral representation formula for functions in $\mathcal{V}_{p}(\lambda)$, i.e., each function in $\mathcal{V}_{p}(\lambda)$ can be expressed as:

$$
\frac{z}{f(z)}=1-\left(\frac{f^{\prime \prime}(0)}{2}\right) z+\lambda z \int_{0}^{z} w(t) d t
$$

where $w \in \mathcal{B}$. Since each $f \in \mathcal{V}_{p}(\lambda)$ is analytic in the disc $\mathbb{D}_{p}:=\{z:|z|<p\}$, therefore it has the Taylor expansion of the form (1.1) valid in $\mathbb{D}_{p}$. In [4, Theorem 5], the authors of the present article established the exact region of variability of the second Taylor coefficients $a_{2}(f), f \in \mathcal{V}_{p}(\lambda)$ which we state below:

$$
\left|a_{2}(f)-1 / p\right| \leq \lambda p,
$$

and made the following conjecture about the exact bounds for the modulus of the $n$-th Taylor coefficients: 
Conjecture 1. If $f \in \mathcal{V}_{p}(\lambda)$ for some $0<\lambda \leq 1$ and has the expansion of the form (1.1) in $\mathbb{D}_{p}$. Then

$$
\left|a_{n}(f)\right| \leq \frac{1-\left(\lambda p^{2}\right)^{n}}{p^{n-1}\left(1-\lambda p^{2}\right)}
$$

for $n \geq 3$ and equality occurs in the above inequality for the following functions:

$$
k_{p}^{\lambda}(z):=\frac{-p z}{(z-p)(1-\lambda p z)} .
$$

Remark. It is easy to check that as $p \rightarrow 1-$, the inequality (1.3) gives the conjectured bound $\left|a_{n}(f)\right| \leq \sum_{k=0}^{n-1} \lambda^{k}, n \geq 3$ for the class $\mathcal{U}(\lambda)$ (see [8]) and for $\lambda=1$ the above conjecture reduces to the Jenkin's theorem (compare [7]) for the class $\Sigma(p)$. Also by taking $p \rightarrow 1-$ and $\lambda=1$ in (1.3) , we will get the famous de Branges theorem for the class $\mathcal{S}$ (compare [5]).

We organise this article as follows. First we prove a representation formula for functions in the class $\mathcal{V}_{p}(\lambda)$. Next, with the help of this representation formula we prove the Conjecture 1 for $n=3,4,5$ with certain range of values of $p$. Finally, we obtain non sharp bounds for $\left|a_{n}(f)\right|, n \geq 3$ and for $\left|a_{n+1}(f)-a_{n}(f) / p\right|, n \geq 2$.

\section{Main Results}

We start this section with the following representation formula for functions in the class $\mathcal{V}_{p}(\lambda)$ :

Theorem 1. Each $f \in \mathcal{V}_{p}(\lambda)$ can be represented as

$$
f(z)=\frac{-p z}{(z-p)(1-\lambda p z w(z))}, z \in \mathbb{D},
$$

where $w \in \mathcal{B}$. Also every $f \in \mathcal{V}_{p}(\lambda)$ can be expressed as

$$
f(z)=\frac{-p z u(z)}{(z-p)(1-\lambda p)}, z \in \mathbb{D},
$$

where $u \in \mathcal{B}$ and $u(0)=1-\lambda p$.

Proof. For every $f \in \mathcal{V}_{p}(\lambda)$ we have from (1.2),

$$
\frac{z}{f(z)}=1-\left(\frac{f^{\prime \prime}(0)}{2}\right) z+\lambda z \int_{0}^{z} w_{1}(t) d t, \quad z \in \mathbb{D},
$$

where $w_{1} \in \mathcal{B}$. Since $f(p)=\infty$, the above equality yields

$$
\frac{f^{\prime \prime}(0)}{2}=\frac{1}{p}\left(1+\lambda p \int_{0}^{p} w_{1}(t) d t\right)
$$

and hence

$$
\frac{z}{f(z)}=1-\frac{z}{p}\left(1+\lambda p \int_{0}^{p} w_{1}(t) d t\right)+\lambda z \int_{0}^{z} w_{1}(t) d t, \quad z \in \mathbb{D} .
$$


Let us define

$$
w(z):=\left(\int_{p}^{z} w_{1}(t) d t\right) /(z-p), z \in \mathbb{D} .
$$

Now it is a simple exercise to see that $|w(z)| \leq 1$ and $w(p)=w_{1}(p)$. Consequently (2.3) takes the following form:

$$
\frac{z}{f(z)}=\frac{-(z-p)(1-\lambda p z w(z))}{p},
$$

where $w \in \mathcal{B}$. This proves the representation formula (2.1). Next we see that

$$
|1-\lambda p z w(z)| \geq 1-\lambda p|z w(z)| \geq 1-\lambda p .
$$

We now define

$$
u(z):=\frac{(1-\lambda p)}{(1-\lambda p z w(z))}, z \in \mathbb{D} .
$$

Then clearly $|u(z)| \leq 1$ and $u(0)=1-\lambda p$. Now plugging (2.4) in (2.1) we get (2.2). This completes the proof of Theorem 1 .

The next theorem deals with the estimate of $\left|a_{n}(f)\right|$ for $n=3,4,5$ under some restriction on the range of values of $p$ where $a_{n}(f)$ is defined by (1.1).

Theorem 2. Let $f \in \mathcal{V}_{p}(\lambda)$ have expansion of the form (1.1). Then the inequality (1.3) holds for $n=3, p \in(0,1 / 2]$; for $n=4, p \in(0,(\sqrt{3}-1) / 2]$ and for $n=5, p \in$ $(0,(\sqrt{5}-1) / 4]$. Equality holds in the above inequality for the function (1.4).

Proof. Let each $w \in \mathcal{B}$ has the following Taylor expansion in $\mathbb{D}$ :

$$
w(z)=\sum_{n=0}^{\infty} c_{n} z^{n}
$$

Now inserting the above expression for $w$ and the series expansion (1.1) for $f$ in the representation formula (2.1) we get,

$$
z+\sum_{n=2}^{\infty} a_{n}(f) z^{n}=z(1-z / p)^{-1}\left(1-\lambda p z \sum_{n=0}^{\infty} c_{n} z^{n}\right)^{-1} .
$$

Next comparing the coefficients of $z^{n}, n=3,4,5$ in the above equality we get

$(2.5) a_{3}(f)=\lambda p c_{1}+\lambda^{2} p^{2} c_{0}^{2}+\lambda c_{0}+1 / p^{2}$,

(2.6) $a_{4}(f)=\lambda p c_{2}+2 \lambda^{2} p^{2} c_{0} c_{1}+\lambda^{3} p^{3} c_{0}^{3}+\lambda c_{1}+\lambda^{2} p c_{0}^{2}+\lambda c_{0} / p+1 / p^{3}$ and

(2.7) $a_{5}(f)=\lambda p c_{3}+\lambda^{2} p^{2} c_{1}^{2}+2 c_{0} c_{2} \lambda^{2} p^{2}+3 c_{0}^{2} c_{1} \lambda^{3} p^{3}+\lambda^{4} p^{4} c_{0}^{4}+\lambda c_{2}$

$$
+2 c_{0} c_{1} \lambda^{2} p+\lambda^{3} p^{2} c_{0}^{3}+\lambda c_{1} / p+\lambda^{2} c_{0}^{2}+\lambda c_{0} / p^{2}+1 / p^{4} .
$$

Now from [13], we know that

$$
\left|c_{0}\right| \leq 1 \text { and }\left|c_{n}\right| \leq 1-\left|c_{0}\right|^{2} \quad \text { for all } n \geq 1 .
$$


Using these inequalities in (2.5) we have

$$
\begin{aligned}
\left|a_{3}(f)\right| & \leq \lambda p\left|c_{1}\right|+\lambda^{2} p^{2}\left|c_{0}\right|^{2}+\lambda\left|c_{0}\right|+1 / p^{2} \\
& \leq \lambda p\left(1-\left|c_{0}\right|^{2}\right)+\lambda\left|c_{0}\right|+\lambda^{2} p^{2}\left|c_{0}\right|^{2}+1 / p^{2} .
\end{aligned}
$$

Setting $\left|c_{0}\right|=x$, we consider $h(x):=\lambda p\left(1-x^{2}\right)+\lambda x+\lambda^{2} p^{2} x^{2}+1 / p^{2}$. So $x \in[0,1]$ and

$$
h^{\prime}(x)=\lambda(1-2 p x)+2 \lambda^{2} p^{2} x .
$$

Now $h^{\prime}(x) \geq 0$ for $p \in(0,1 / 2]$ and for $0<\lambda \leq 1$. This shows that the function $h$ is increasing in $[0,1]$. Therefore,

$$
\max _{0 \leq x \leq 1} h(x)=h(1)=\lambda+\lambda^{2} p^{2}+1 / p^{2}
$$

and thus

$$
\left|a_{3}(f)\right| \leq 1 / p^{2}+\lambda+\lambda^{2} p^{2}=\left(1-\left(\lambda p^{2}\right)^{3}\right) / p^{2}\left(1-\lambda p^{2}\right) .
$$

Using this similar idea, now we prove the conjectured bound for $\left|a_{4}(f)\right|$ and $\left|a_{5}(f)\right|$ when $p$ lies in the intervals stated in the theorem. If we use triangle inequality and the bounds for $\left|c_{n}\right|, n \geq 1$ in (2.6), we get

$$
\begin{aligned}
\left|a_{4}(f)\right| \leq & \left(-2 \lambda^{2} p^{2}+\lambda^{3} p^{3}\right)\left|c_{0}\right|^{3}+\left(-\lambda p-\lambda+\lambda^{2} p\right)\left|c_{0}\right|^{2} \\
& +\left(2 \lambda^{2} p^{2}+\lambda / p\right)\left|c_{0}\right|+\lambda p+\lambda+1 / p^{3} .
\end{aligned}
$$

As before setting $\left|c_{0}\right|=x$, we introduce the following function:

$g(x):=\left(-2 \lambda^{2} p^{2}+\lambda^{3} p^{3}\right) x^{3}+\left(-\lambda p-\lambda+\lambda^{2} p\right) x^{2}+\left(2 \lambda^{2} p^{2}+\lambda / p\right) x+\lambda p+\lambda+1 / p^{3}$.

Since $\left(-2 \lambda^{2} p^{2}+\lambda^{3} p^{3}\right)<0$ and $\left(-\lambda p-\lambda+\lambda^{2} p\right)<0$, then we have

$$
\begin{aligned}
g^{\prime}(x) & =3\left(-2 \lambda^{2} p^{2}+\lambda^{3} p^{3}\right) x^{2}+2\left(-\lambda p-\lambda+\lambda^{2} p\right) x+2 \lambda^{2} p^{2}+\lambda / p \\
& \geq 3\left(-2 \lambda^{2} p^{2}+\lambda^{3} p^{3}\right)+2\left(-\lambda p-\lambda+\lambda^{2} p\right)+2 \lambda^{2} p^{2}+\lambda / p \\
& =-4 \lambda^{2} p^{2}+3 \lambda^{3} p^{3}-2 \lambda p-2 \lambda+2 \lambda^{2} p+\lambda / p \\
& =3 \lambda^{3} p^{3}+2 \lambda^{2} p(1-2 p)+\lambda(1 / p-2-2 p) .
\end{aligned}
$$

Now it is a simple exercise to check that $(1-2 p)>0$ and $(1 / p-2-2 p)>0$ for $p \in(0,(\sqrt{3}-1) / 2]$. Hence $g^{\prime}(x) \geq 0$ for all $0 \leq x \leq 1$ and $p \in(0,(\sqrt{3}-1) / 2]$. This implies that $g$ is increasing in $[0,1]$ and

$$
\max _{0 \leq x \leq 1} g(x)=g(1)=\left(1+\lambda p^{2}+\lambda^{2} p^{4}+\lambda^{3} p^{6}\right) / p^{3} .
$$

This settles our claim for the bound of the coefficients $a_{4}(f)$. Finally we turn our attention into proving the coefficient bound of $\left|a_{5}(f)\right|$. Use of the estimate (2.8) in (2.7) gives

$$
\begin{aligned}
\left|a_{5}(f)\right| \leq & \left(\lambda^{4} p^{4}+\lambda^{2} p^{2}-3 \lambda^{3} p^{3}\right)\left|c_{0}\right|^{4}+\left(-2 \lambda^{2} p^{2}-2 \lambda^{2} p+\lambda^{3} p^{2}\right)\left|c_{0}\right|^{3} \\
& +\left(-\lambda p-2 \lambda^{2} p^{2}+3 \lambda^{3} p^{3}-\lambda+\lambda^{2}-\lambda / p\right)\left|c_{0}\right|^{2} \\
& +\left(2 \lambda^{2} p^{2}+2 \lambda^{2} p+\lambda / p^{2}\right)\left|c_{0}\right|+\lambda p+\lambda^{2} p^{2}+\lambda+\lambda / p+1 / p^{4} .
\end{aligned}
$$


Now assume as before $\left|c_{0}\right|=x \in[0,1]$ and define

$$
\begin{aligned}
q(x):= & \left(\lambda^{4} p^{4}+\lambda^{2} p^{2}-3 \lambda^{3} p^{3}\right) x^{4}+\left(-2 \lambda^{2} p^{2}-2 \lambda^{2} p+\lambda^{3} p^{2}\right) x^{3} \\
& +\left(-\lambda p-2 \lambda^{2} p^{2}+3 \lambda^{3} p^{3}-\lambda+\lambda^{2}-\lambda / p\right) x^{2} \\
& +\left(2 \lambda^{2} p^{2}+2 \lambda^{2} p+\lambda / p^{2}\right) x+\lambda p+\lambda^{2} p^{2}+\lambda+\lambda / p+1 / p^{4} .
\end{aligned}
$$

Thus we have

$$
\begin{aligned}
q^{\prime}(x)= & 4\left(\lambda^{4} p^{4}+\lambda^{2} p^{2}-3 \lambda^{3} p^{3}\right) x^{3}+3\left(-2 \lambda^{2} p^{2}-2 \lambda^{2} p+\lambda^{3} p^{2}\right) x^{2} \\
& +2\left(-\lambda p-2 \lambda^{2} p^{2}+3 \lambda^{3} p^{3}-\lambda+\lambda^{2}-\lambda / p\right) x+2 \lambda^{2} p^{2}+2 \lambda^{2} p+\lambda / p^{2} .
\end{aligned}
$$

Since $\left(\lambda^{4} p^{4}+\lambda^{2} p^{2}\right) \geq 2\left(\lambda^{6} p^{6}\right)^{1 / 2}$ and both the quantities $\left(-2 \lambda^{2} p^{2}-2 \lambda^{2} p+\lambda^{3} p^{2}\right)$ and $\left(-\lambda p-2 \lambda^{2} p^{2}+3 \lambda^{3} p^{3}-\lambda+\lambda^{2}-\lambda / p\right)$ are negative, so

$$
\begin{aligned}
q^{\prime}(x) & \geq 2 \lambda^{3} p^{3}-8 \lambda^{2} p^{2}-4 \lambda^{2} p+3 \lambda^{3} p^{2}-2 \lambda p-2 \lambda-2 \lambda / p+2 \lambda^{2}+\lambda / p^{2} \\
& =2 \lambda^{3} p^{3}+3 \lambda^{3} p^{2}-2 \lambda^{2}\left(4 p^{2}+2 p-1\right)-\lambda\left(2 p+2+2 / p-1 / p^{2}\right) .
\end{aligned}
$$

Again since $\left(4 p^{2}+2 p-1\right)<0$ and $\left(2 p+2+2 / p-1 / p^{2}\right)<0$ for $p \in(0,(\sqrt{5}-1) / 4]$, the above inequality gives $q^{\prime}(x) \geq 0$ i.e. , the function $q$ is increasing in the interval $[0,1]$ and hence

$$
\max _{0 \leq x \leq 1} q(x)=q(1)=\left(1+\lambda p^{2}+\lambda^{2} p^{4}+\lambda^{3} p^{6}+\lambda^{4} p^{8}\right) / p^{4} .
$$

This proves our claim regarding the coefficient bound of $a_{5}(f)$. Also it is easy to check that

$$
k_{p}^{\lambda}(z)=\sum_{n=1}^{\infty} \frac{1-\lambda^{n} p^{2 n}}{p^{n-1}\left(1-\lambda p^{2}\right)} z^{n}, \quad z \in \mathbb{D}_{p} .
$$

This proves the sharpness part of the inequality stated in the theorem.

Remark. Using similar lines of proof as in the previous theorem one may be able to prove the conjectured bound for $n \geq 6$ with certain range of values of $p$. However, we expect that this will need much more effort in calculation. We leave this open to the interested reader.

Here we present the following definition which we need for our further discussion.

Definition 2.1. For any two analytic functions $f$ and $g, f$ is said to be subordinate to the function $g$ if the relation $f(z)=g(w(z)), z \in \mathbb{D}$ holds for any $w \in \mathcal{B}$ with $w(0)=0$. This phenomenon is abbreviated as $f \prec g$.

In the next result we prove non sharp bounds for the absolute value of the Taylor coefficients $\left|a_{n}(f)\right|$ for $n \geq 3$ and $\left|a_{n+1}(f)-a_{n}(f) / p\right|$ for $n \geq 2$ whenever $f \in \mathcal{V}_{p}(\lambda)$.

Theorem 3. Let $f \in \mathcal{V}_{p}(\lambda)$ be of the form (1.1) in $\mathbb{D}_{p}$. Then for $n \geq 3$,

$$
\left|a_{n}(f)\right| \leq \frac{1}{p^{n-1}}+\left(\sum_{k=1}^{n-1} \lambda^{2 k} p^{2 k}\right)^{1 / 2}\left(\sum_{k=1}^{n-1} \frac{1}{p^{2(n-k-1)}}\right)^{1 / 2}
$$

and for $n \geq 2$, we have

$$
\left|a_{n+1}(f)-a_{n}(f) / p\right| \leq \lambda p \text {. }
$$


Proof. From the representation (2.1) we see that

$$
f(z)=f_{1}(z) f_{2}(z), \quad z \in \mathbb{D},
$$

where

$$
f_{1}(z):=\frac{-p z}{(z-p)} \quad \text { and } \quad f_{2}(z):=\frac{1}{(1-\lambda p z w(z))}, w \in \mathcal{B} .
$$

Let us now consider the Taylor expansions for the following functions in $\mathbb{D}$ :

$$
f_{1}(z)=\sum_{n=1}^{\infty} \frac{1}{p^{n-1}} z^{n}:=\sum_{n=1}^{\infty} A_{n} z^{n}, f_{2}(z)=\sum_{n=0}^{\infty} B_{n} z^{n} \text { and } \quad 1 /(1-\lambda p z)=\sum_{n=0}^{\infty}(\lambda p)^{n} z^{n} .
$$

Since $f_{2} \prec 1 /(1-\lambda p z)$, therefore using Rogosinski's theorem [12, Theorem II], we get

$$
\sum_{k=1}^{n-1}\left|B_{k}\right|^{2} \leq \sum_{k=1}^{n-1} \lambda^{2 k} p^{2 k}
$$

Now equating coefficients of $z^{n}$ on both sides of the equation (2.10), we get

$$
a_{n}(f)=A_{n}+\sum_{k=1}^{n-1} B_{k} A_{n-k}
$$

where $A_{n}=1 / p^{n-1}$. Thus an application of the Cauchy-Schwarz inequality yields

$$
\begin{aligned}
\left|a_{n}(f)\right| & \leq\left|A_{n}\right|+\left|\sum_{k=1}^{n-1} B_{k} A_{n-k}\right| \\
& \leq\left|A_{n}\right|+\left(\sum_{k=1}^{n-1}\left|B_{k}\right|^{2}\right)^{1 / 2}\left(\sum_{k=1}^{n-1}\left|A_{n-k}\right|^{2}\right)^{1 / 2} \\
& \left.\leq \frac{1}{p^{n-1}}+\left(\sum_{k=1}^{n-1} \lambda^{2 k} p^{2 k}\right)^{1 / 2}\left(\sum_{k=1}^{n-1} \frac{1}{p^{2(n-k-1)}}\right)^{1 / 2} \quad \text { (by (2.11) }\right)
\end{aligned}
$$

which is the required bound that we wish to prove. Again using the representation (2.1) in Theorem 1, we see that $(z-p) f(z) /(-p z) \prec 1 /(1-\lambda p z)=\sum_{n=0}^{\infty}(\lambda p)^{n} z^{n}$. Now since $f$ has expansion of the form (1.1), we compute

$$
\frac{(z-p) f(z)}{-p z}=1+\sum_{n=1}^{\infty}\left(a_{n+1}(f)-a_{n}(f) / p\right) z^{n} .
$$

Next we note that the sequence $\left\{(\lambda p)^{n}\right\}$ is a nonnegative, decreasing and convex sequence. Here we clarify that a real sequence $\left\{x_{n}\right\}$ is called convex sequence if $x_{n-1}+x_{n+1} \geq 2 x_{n}$ holds for all $n$. Now an application of a well-known result of Rogosinski (see [12, Theorem VII]), we have

$$
\left|a_{n+1}(f)-a_{n}(f) / p\right| \leq \lambda p, \quad \forall n \geq 2 .
$$

This completes the proof of the theorem. 
Remark. In [11], the authors proved the following non sharp bound for $\left|a_{n}(f)\right|, n \geq 3$ for functions in the class $\mathcal{U}(\lambda)$ :

$$
\left|a_{n}(f)\right| \leq 1+\lambda \sqrt{n-1} \sqrt{\sum_{k=0}^{n-2} \lambda^{2 k}} .
$$

We see that as $p \rightarrow 1-$, the obtained bound (2.9) coincides with the above bound.

\section{REFERENCES}

[1] L. A. Aksentév, Sufficient conditions for univalence of regular functions (Russian), Izv. Vyš̌. Učebn. Zaved. Matematika, 1958(4) (1958), 3-7.

[2] B. Bhowmik and F. Parveen, On a subclass of meromorphic univalent functions, Complex Var. Elliptic Equ., 62 (2017), 494-510.

[3] B. Bhowmik and F. Parveen, Criteria for univalence, integral means and dirichlet integral for meromorphic functions, Bull. Belg. Math. Soc.(Simon Stevin), 24 (2017), 427-438.

[4] B. Bhowmik and F. Parveen, Sufficient conditions for univalence and study of a class of meromorphic univalent functions, Bull. Korean Math. Soc., To appear, arXiv:1705.06009,

[5] L. De Branges, A proof of the Bieberbach conjecture, Acta Math., 154(1985), 137-152.

[6] R. Fournier and S. Ponnusamy, A class of locally univalent functions defined by a differential inequality, Complex Var. Elliptic Equ., 52(1)(2007), 1-8.

[7] J.A. Jenkins, On a conjecture of Goodman concerning meromorphic univalent function, Michigan Math. J., 9 (1962), 25-27.

[8] M. Obradović, S. Ponnusamy and K.-J. Wirths, Geometric studies on the class $\mathcal{U}(\lambda)$, Bull. Malays. Math. Sci. Soc., 39(2016), 1259-1284.

[9] M. Obradović, S. Ponnusamy and K.-J. Wirths, Logarithmic coefficients and a coefficient conjecture for univalent functions, Monatsh Math., To appear.

[10] M. Obradović and S. Ponnusamy, Univalence and starlikeness of certain integral transforms defined by convolution of analytic functions, J. Math. Anal. Appl., 336(2007), 758-767.

[11] S. Ponnusamy and K.-J. Wirths, Coefficient problems on the class $\mathcal{U}(\lambda)$, Preprint, arXiv:1709.06336,

[12] W. Rogosinski, On the coefficientc of subordinate functions, Proc. London Math. Soc., 48(1943), 48-82.

[13] St. Ruscheweyh, Two remarks on bounded analytic functions, Serdica, 11(1985), 200-202.

Bappaditya Bhowmik, Department of Mathematics, Indian Institute of TechnolOGY KharagPur, KharagPur - 721302, India.

E-mail address: bappaditya@maths.iitkgp.ernet.in

Firdoshi Parveen, Department of Mathematics, Indian Institute of Technology KharagPUR, KHARAGPUR - 721302, INDIA.

E-mail address: frd.par@maths.iitkgp.ernet.in 